ist an der Luft aber äusserst zerfliesslich und unterscheidet sich dadurch wesentlich von dem salzsauren Tetramethylaldin, das schön krystallisirt und völlig luftbeständig ist. Das Platimdoppelsalz dieses salzsauren Salzes wurde in derben, dunkelrothen Krystallen gewonnen. Die Untersuchung dieser Körper ist noch im Gang, zeigt aber jetzt schon, dass auch die Aldine der Fettreihe nicht direct durch Reduction der Isonitrosoketone entstehen, wean in saurer Lösung gearbeitet wird. Hierbei entstehen vielmehr Salze, die von den Salzen der Aldine verschieden sind und erst durch Zersetzung mittelst Ammoniak die Aldine liefern.

Wir beabsichtigen unsere Versuche über die Reduction der Nitrosoketone der Fettreihe fortzusetzen und hoffen demnächst weitẹr über dieselben berichten zu können. Auch die Reduction des Benzilmonoxims, die, in alkalischer Lösung vorgenommen, uns das Tetraphenylaldin lieferte, soll in saurer Lösung wiederholt werden.

Göttingen. Universitäts-Laboratorium.

233. Hercules Tornöe: Ueber das Trimethylen und die Bildung des Allylalkohols aus symmetrischem Dichlorhydrin.

(Eingegangen am 3. April.)

Die von Hübner und Müller ${ }^{1}$ ) beobachtete Bildung des gewöhnlichen Allylalkohols aus symmetrischem Dichlorhydrin und Natrium in ätherischer Lösuug hat sich von jeher nicht in einfacher Weise erklären lassen und kann sowohl mit mehreren anderen Thatsachen wie auch mit den herrschenden Anschaungen über die Constitution der Dichlorhydrine zur Zeit nicht in Einklang gebracht werden. Solange nur der misslungene Versuch Reboul's, das Trimethylen zu gewinnen, bekannt war, glaubte man die Ursache der von $H u ̈ b n e r$ und Müller beobachteten Umlagerung in der Nichtexistenzfähigkeit der Isomeren des Propylens und des Allylalkohols erblicken zu können. Mit dieser Annahme hatte man allerdings eine Erklärung für die Unmöglichkeit, den isomeren Allylalkohol zu erhalten, die Bildung des gewöhnlichen Allylalkohols bedurfte aber immerhin einer weiteren Erklärung. Als nun das Trimethylen zuerst von Freund ${ }^{2}$ ) and später von Gustavson ${ }^{3}$ ) dargestellt wurde und

1) Ann. Chem. Pharm. 159, 168.

2) Journ. für prakt. Chem. [2] XXVI, 367.

3) Journ. für prakt. Chem. [2] XXXVI, 300. 
sich als ein beständiger, wohl charakterisirter Körper erwies, schien die Annahme von der Nichtexistenzfähigkeit des isomeren Allylalkohols weit weniger wahrscheinlich. Die einzige Stütze derselben lag nun lediglich in der Unmöglichkeit, den isomeren Allylalkohol bei dem Versuch zu erhalten, der von Hübner und Müller angestellt and bei dem seine Bildung zu erwarten war.

In Anbetracht der Wichtigkeit der genannten Reaction für die Beurtheilung der Constitution der Dichlorhydrine beschloss ich auf Veranlassung von Hrn. Prof. V. Meyer, die Frage wieder aufzunehmen in der Hoffnung, den Mechanismus der Reaction aufklären zu können.

Ehe ich an die eigentlichen Versuche herantrat, habe ich nach der von Gustavson angegebenen Methode das Trimethylen dargestellt, um die Mittheilnngen von Freund und Gustavson durch eine Bestimmung der Dampfdichte zu ergänzen. Auch schien es wünschenswerth, eine wirkliche Analyse des Gases auszuführen, indem die genannten Forscher sich in dieser Beziehung mit einer Bestimmung des Verhältnisses von Kohlenstoff and Wasserstoff begnügt haben. Dieser doppelte Zweck lässt sich leicht folgendermassen mittelst einer einzigen Operation erreichen.

\section{Ueber Trimethylen.}

Nachdem die für die Darstellung nöthigen Mengen Trimethylenbromid, Zinkstaub und Alkohol in ein Gasentwickelungskölbchen gebracht waren, wurde aus demselben und aus dem vorgelegten Waschapparat die Luft vollständig mittelst reiner Kohlensäure vertrieben. Noch während des Ausströmens der Koblensäure wurde die Verbindung mit dem völlig mit Wasser gefüllten Gasometer hergestellt.

Kurzes Erwärmen des Entwickelungskölbchens genügte, um die Reaction einzuleiten; sie geht dann von selbst weiter, indem man nur gegen Ende dieselbe nochmals durch Erwärmen unterstützen muss. Zur Reinigung wurde das Gas durch ein kleines, in einer Kältemischung befindliches U-Rohr geleitet, welches Alkohol enthielt. Für die Abwägung sowie für die Bestimmung der Dampfdichte des Gases diente ein beiderseits mit Hähnen versehenes Glassgefäss, dessen inneres Volumen durch Wägung mit Wasser zu $178.5 \mathrm{ccm}$ bestimmt worden war. In allen Fällen, wo dasselbe mit Luft, Gas oder Kohlensäure gewogen wurde, war auf der andern Waagschale ein ähnliches zugeschmolzenes Glassgefäss von demselben äusseren Volum angebracht, wodurch die Nothwendigkeit einer Reduction der Wägungen auf den luftleeren Raum umgangen wurde.

Bei der Ueberfüllung des Gases aus dem Gasometer in das Glasgefäss wurde dasselbe Prinzip wie bei der Entwickelung befolgt. 
Durch das Gefäss, sowie ein damit verbundenes Chlorcalciumroh strich getrocknete Kohlensäure, bis die Luft völlig vertrieben war Dann wurde das Gas aus dem Gasometer durch das Chlorcalciumroh: in das Gefäss geleitet, bis der grösste Theil der Kohlensäure ver drängt war. Die Hähne wurden darauf geschlossen, Temperatur un Druck abgelesen und der Apparat gewogen. Durch Wägung mi trockener Luft von bekannter Temperatur und Druck war das Ge wicht des luftleeren Gefässes schon im voraus ermittelt worden, E; berechnet sich hieraus mit Leichtigkeit die Summe der Gewichte de: eingeschlossenen Gases und der demselben beigemischten Kohlensäure

Bei der Verbrennung, die im übrigen in der üblichen Weise ge schah, wurde das Gas mittelst Luft aus dem Glasgefäss durch eir gewogenes, mit Natronkalk und Chlorcalcium gefülltes Robr in das Verbrennungsrohr hinübergetrieben. Die Differenz aus der Gewicht. zunahme des vorgelegten Natronkalkrohres und der früher bestimmter Summe der Gewichte des Gases und der Kohlensäure gab das Ge. wicht der verbrannten Gasmenge.

Wie leicht zu ersehen ist, genügen die angestellten Beobachtungen auch, um das specifische Gewicht des Gases zu berechnen. Ein bei der Bestimmung der beigemengten Kohlensäure begangener Fehler kann das Resultat der Dampfdichtebestimmung nur wenig beeinflussen. da die Dichte des Gases sich derjenigen der Kohlensäure sehr nähert. Bei der Einfachheit sowohl des Prinzips des Verfahrens wie der Berechnung der Resultate brauche ich nicht auf Einzelheiten einzugehen. Die Resultate der Analysen und Dichtebestimmungen waren folgende:

I. $0.2034 \mathrm{~g}$ Gas gaben $0.6257 \mathrm{~g}$ Kohlensäure und $0.2563 \mathrm{~g}$ Wasser.

II. $0.1718 \mathrm{~g}$ Gas gaben $0.5404 \mathrm{~g}$ Kohlensäure und $0.2246 \mathrm{~g}$ Wasser.

\begin{tabular}{cccc} 
& \multicolumn{2}{c}{ Gefunden } & Ber. für $\mathrm{C}_{3} \mathrm{H}_{6}$ \\
& I. & II. & $85.7 \mathrm{pCt}$. \\
$\mathrm{C}$ & 83.9 & 85.8 & $14.3 \%$ \\
$\mathrm{H}$ & 14.0 & 14.5 & \\
& \multicolumn{3}{c}{ Dichtebestimmung. } \\
& \multicolumn{3}{c}{ Gefunden } \\
& I. & II. & Ber. für $\mathrm{C}_{3} \mathrm{H}_{6}$ \\
d & 1.468 & 1.461 & 1,455
\end{tabular}

Das Gas war für jede Analyse neu dargestellt. Die wenig gute Uebereinstimmung der Zahlen der ersten Analyse mit der Theorie kann kaum einer Verunreinigung zugeschrieben werden, indem Kohlenstoff und Wasserstoff in dem richtigen Verhältniss zu einander stehen. Wahrscheinlich rührt der Fehler daher, dass sich bei der Ueberfüllung des Gases etwas Luft beigemengt hat; auch darf man bei einer derartigen Analyse mit so zahlreichen Fehlerquellen wohl kaum erwarten, den gewöhnlichen Ansprüchen in Bezug auf Genauigkeit der Resultate völlig genügen zu können. 
Da ich das Gas einmal unter Händen hatte, behandelte ich dasselbe auch mit Brom und Jodwasserstoff, um mich durch eigene Anschaung davon zu überzeugen, wie verschieden diese Agentien auf das Trimethylen und auf das Propylen wirken. Der Unterschied des Verhaltens des Trimethylens von demjenigen des Propylens, besonders gegenüber Brom, ist von Freund und Gustavson sicher nicht übertrieben worden, ich wäre vielmehr geneigt, demselben noch einen etwas kräftigeren Ausdruck zu verleihen.

Aus der oben angegebenen Zusammensetzung des Gases in Verbindung mit der gleichzeitig festgestellten Dampfdichte geht hervor, dass dem Trimethylen die Formel $\mathrm{C}_{3} \mathrm{H}_{6}$ zukommt; ferner ist von Freund und Gustavson mit Sicherheit nachgewiesen worden, dass dasselbe mit dem Propylen nicht identisch sein kann. Durch diese Thatsachen ist der Körper genügend charakterisirt.

\section{Ueber Dichlorhydrin.}

Die von Hübner und Müller beobachtete Wirkung von Natrium auf symmetrisches Dichlorhydrin in ätherischer Lösung habe ich bei der Wichtigkeit dieser Reaction mit grösster Sorgfalt von neuem untersucht. Das für meine Untersuchungen dienende Dichlorhydrin hatte ich selbst aus reinem Epichlorhydrin vom Siedepunkt 113.8 ${ }^{\circ}$ ) dargestellt.

Das Dichlorhydrin siedete zwischen $173.5^{\circ}$ und $175.5^{\circ}$, und die weit überwiegende Menge ging fast constant bei $174.3^{\circ}$ (Barometer $760.5 \mathrm{~mm}$ ) über.

Häbner und Müller geben an, dass die Einwirkung des Natriums auf das Dichlorhydrin bei ihren Versuchen so beftig war, dass beträchtliche Mengen Aether sich verflüchtigten. Durch energische Abkühlung, indem ich das Gefäss in einem Brei von Schnee und rauchender Salzsäure heftig umschüttelte, ist es mir mit einer einzigen Ausnahme gelungen, die Temperatur des Reactionsgemisches unter dem Siedepunkt des Aethers zu halten. Bei meinen Versuchen wurden immer $50 \mathrm{~g}$ Dichlorhydrin in $150-200 \mathrm{~g}$ absolutem Aether gelöst und mit der berechneten Menge Natrium auf ein Mal zusammengebracht.

1) Alle in dieser Abhandlung angeführten Temperaturbestimmungen sind mittelst eines Thermometers gemacht, dessen Fundamentabstand und Caliberfehler ich selbst bestimmt hatte. Die Summe der bezüglichen Correctionen war für die in Frage kommenden Temperaturen mit einer Genauigkeit von etwa $0.2^{\circ}$ bekannt. Wo nicht der ganze Quecksilberfaden auf die betreffende Temperatur erhitzt war, ist die Poggendorff'sche Correction angebracht worden. Die Correctionen, welche die so verbesserten Zahlen auf Ergebnisse des Luftthermometers reduciren sollten, waren leider nicht mit genügender Genauigkeit bekannt und sind in Folge dessen nicht angebracht. 
Es muss nämlich immer Dichlorhydrin in Ueberschuss vorhanden sein, da die Reaction sonst in anderem Sinne verläuft unter Bildung grösserer Mengen hochsiedender Condensationsproducte. Nachdem die heftigste Einwirkung vorüber war, warde das Gemisch einige Stunden sich selbst überlassen, wobei das Natrium vollständig verschwand. Nachher wurde so viel $\mathrm{W}$ asser zugesetzt, dass das gebildete Chlornatrium sich eben löste. Die zwei Schichten wurden getrennt und von der wässerigen Schicht so viel abdestillirt, bis kein Alkohol mehr überging. Ans dem Destillat wurde der Alkohol mittelst Pottasche abgeschieden. Die ätherische Schicht wurde mit calcinirter Pottasche getrocknet und mittelst eines Lin nemann'schen Apparats der fractionirten Destillation unterworfen. Ich gewann in dieser Weise aus $100 \mathrm{~g}$ Dichlorhydrin $23 \mathrm{~g}$ fast reinen Alkohols, das heisst etwa $50 \mathrm{pCt}$. der theoretischen Ausbeute; hochsiedende Condensationsproducte traten nur in geringer Menge auf. Während der Reaction entwich fortwährend ein Gas, das ich nicht habe von den Aetherdämpfen trennen und analysiren können. Der so dargestellte Alkohol ist nicht ganz chlorfrei, und die, wie es scheint, ziemlich flüchtige, chlorhaltige Verunreinigung lässt sich schwerlich durch fractionirte Destillation vollständig entfernen. Bis jetzt habe ich diesen chlorhaltigen Körper leider noch nicht fassen können. Ganz chlorfrei erhält man den Alkohol, wenn man denselben mit wenig festem Kalihydrat im Rohr kurze Zeit auf etwa $70^{\circ}$ erhitzt. Bei dieser Operation bildet sich ein bei $227-229^{\circ}$ siedendes Oel, dessen Zusammensetzung der Formel $\left(\mathrm{C}_{10} \mathrm{H}_{18} \mathrm{O}_{4}\right) \mathrm{n}$ entspricht. Eine bei derselben Temperatur siedende Flüssigkeit (wahrscheinlich dieselbe) findet sich auch unter den bochsiedenden Producten, die sich bei der Hauptreaction bilden. Charakteristisch für das Oel ist, dass dasselbe bei der Destillation entweder durch Zersetzung oder durch Polymerisation einen festen weissen Körper abscheidet, der sowohl die Wände des Fractionirkölbchens als auch die Kugel des Thermometers bedeckt und ein lästiges Stossen der Flüssigkeit hervorruft Die Analyse des Oeles gab folgendes Resultat:

I. $0.1820 \mathrm{~g}$ Substanz gaben $0.3936 \mathrm{~g}$ Kohlensäure und $0.1465 \mathrm{~g}$ Wasser. II. $0.2218 \mathrm{~g}$ Substanz gaben $0.4820 \mathrm{~g}$ Kohlensäure und $0.1785 \mathrm{~g}$ Wasser.

$$
\begin{array}{crcc} 
& \multicolumn{2}{c}{\text { Gefunden }} & \text { Ber. für }\left(\mathrm{C}_{10} \mathrm{H}_{18} \mathrm{O}_{4}\right) \mathrm{D} \\
& \multicolumn{1}{c}{\mathrm{I} .} & \text { II. } & 59.41 \mathrm{pCt} \\
\mathrm{C} & 58.98 & 59.27 & 8.91 》 \\
\mathrm{H} & 8.94 & 8.94 &
\end{array}
$$

Die Menge, die mir von diesem Körper zur Verfügung stand, war zu gering, um eine nähere Untersuchung zu ermöglichen.

Der gereinigte Alkohol lässt sich mittelst Pottasche oder wasserfreiem schwefelsaurem Natron nicht völlig trocken erhalten, vielmehr ist dazu Kalk oder Baryt erforderlich. Es verursachte aber die Anwendung dieser Mittel so grosse Verluste, dass ich vorgezogen babe, 
etwas ron dem Alkohol mittelst Acetylchlorid in den Ester überzuführen, um ihn in dieser Form zu analysiren.

Der gebildete. Ester siedete bei $103-105^{\circ}$, während die früheren Forscher im Durchschnitt $103.5^{\circ}$ als Siedepunkt des gewöhnlichen Essigsäureallylesters angeben.

$0.1640 \mathrm{~g}$ Substan\% gaben $0.3595 \mathrm{~g}$ Kohlensäure und $0.1212 \mathrm{~g}$ Wasser.

$\begin{array}{ccc} & \text { Gofundon } & \text { Berochnet } \\ \mathrm{C} & 59.78 & 60.00 \mathrm{pCt} \\ \mathrm{H} & 8.21 & 8.00\end{array}$

Der Alkohol verhielt sich äusserlich ganz wie der gewöhnliche Allylalkohol, den ich zum Vergleich mit dem anderen aus der Kahlbaum'schen Fabrik bezogen hatte. Besonders charakteristisch ist, wie Tollens und Henninger ${ }^{1}$ ) hervorgehoben haben, der Geruch, indem die unangenehme Wirkung auf die Geruchsorgane sich erst nach einiger Zeit bemerkbar macht.

Cm die Identität meines Alkohols mit dem gewöhnlichen Allylalkohol festzustellen, schien es am zweckmässigsten, denselben in eine leicht zu reinigende, womöglich gut krystallisirende Verbindung überzuführen. Als solche empfahl sich in erster Linie das Thiosinamin, welches ich in der That durch successive Ueberführung des Alkohols in Bromid und Senföl bereitet habe. Die entsprechenden Verbindungen aus gewöhnlichem Allylalkohol wurden zurn Vergleich auch dargestellt. Jch habe mich natürlicherweise auch bei der Darstellung des Bromides and des Senföles bemüht, möglichst reine Producte zu bekommen, um durch die Siedepunkte derselben einen Anhaltspunkt für die Beantwortung der gestellten Frage zu bokommen. Es gelang mir dies anch in unerwartetem Maasse, indem nach beendigter Reinigung die weitaus grössten Mengen sowohl der Bromide als auch der Senföle innerhalb eines Temperaturutervalles von höehstens $0.2^{\circ}$ übergingen. Von den Bromiden siedete das aus gewöhnlichem Allylalkohol dargestellte bei $70.2^{\circ}$ (Barometer $745 \mathrm{~mm}$ ), und das von meinem Alkohol herrührende bei $70.1^{\circ}$ (Barometer $749 \mathrm{~mm}$ ). Für die Senföle wurden die entsprechenden Siedepunkte $151.2^{\circ}$ (Barometer $745 \mathrm{~mm}$ ) und $151.1^{\circ}$ (Barometer $740 \mathrm{~mm}$ ) gefunden. Zwei zn vergleichende Siedepunkte wurden immer in demselben Gefäss bestimmt.

Das Thiosinamin besitzt ein ausgezeichnetes Krystallisationsvermögen und schiesst aus alkoholischer Lösung in wasserhellen, sechsseitigen Tafeln an. Nach früheren Angaben soll die Substanz in Aether leicht löslich sein, ich möchte sie jedoch vielmehr als scliwer löslich in A ether bezeichnen. Beim Schmelzen zeigt das Thiosinamin ein sehr charakteristisches Verhalten, indem sein Pulver bei etwa 710

1) Ann. Chem. Pharm. 156, 137. 
im Capillarrohr zusammensinkt, ohne zu schmelzen; es bleibt dann bei weiterer Temperaturerhöhung anscheinend ganz unverändert, bis es bei $78.4^{\circ}$ plötzlich schmilzt. Wird die entstandene wasserhelle Flüssigkeit im Röhrchen wieder abgekühlt, so erstarrt sie za einer milchglasähnlichen Masse, welche nunmehr völlig scharf bei $78.4^{\circ}$ schmilzt, ohne dass irgend eine vorangehende Veränderung beobachtet werden kann. Alle beide Thiosinamine, sowohl das von gewöhnlichem Allylalkohol als das von meinem Alkohol herrührende, waren prachtvolle Präparate, welche sich beim Schmelzen genau gleich verhielten. Dieses deutlich wahrnehmbare Zusammensinken vor dem Schmelzen musste ursprünglich als ein Zeichen der Unreinbeit des Thiosinamins betrachtet werden, und ich habe deswegen das schon gereinigte Thiosinamin noch fünfmal umkrystallisirt, ohne indessen die geringste Hebung des Schmelzpunktes hervorrufen zu können.

Ausser dem Thiosinamin ist meines Wissens keine andere krystallisirbare Allylverbindung dargestellt worden, man konnte aber voraussehen, dass der Carbaminsäureester analog den anderen Carbaminsäureestern fest und gut krystallisirbar sein würde. Ich habe auch diesen Körper bereitet, indem ich Phosgen in Allylalkohol einleitete und das fractionirte Product mit Ammoniak versetzte. Die wässerige Lösung wurde zur Trockene verdampft und aus dem Rückstande der Ester mittelst Aether ausgezogen. Nach dem Verdunsten des Aethers konnte der Ester durch kurzes Eintauchen in Schnee zum Krystallisiren gebracht werden. Ich gewann ihn durch Abpressen zwischen Fliesspapier in dex Kälte als einen ganz farblosen Körper, der indessen sicherlich nicht in demselben Maasse rein war, wie das Thiosinamin. Eine für genaue, vergleichende Bestimmungen genügende Reinigung lässt sich nicht leicht erreichen, erstens weil der Schmelzpunkt viel tiefer liegt als im Voraus vermuthet wurde, zweitens weil der Ester in den gewöhnlichen Lösungsmitteln so leicht löslich ist, dass er bei gewöhnlicher Temperatur sich nicht aus Lösungen umkrystallisiren lässt; überdies zerfiesst er, kaum getrocknet, schnell an der Luft.

Das Aussehen des Esters ist ziemlich charakteristisch, besonders wenn er aus dem füssigen Zustande langsam in den festen übergeht, wobei er in langen prismatischen Nadeln krystallisirt. Der Carbaminsäureallylester ist analog den anderen Carbaminsäurcestern unzersetzt destillirbar.

I. $0.2175 \mathrm{~g}$ Substanz gaben $24.80 \mathrm{ccm}$ fenchten Stickstoff bei 90 und $755.7 \mathrm{~mm}$ Druek.

II. $0.1735 \mathrm{~g}$ Substanz gaben $20.34 \mathrm{ccm}$ feuchten Stickstoff bei $10^{\circ}$ und $743.5 \mathrm{~mm}$ Druck.

$$
\begin{array}{cccc} 
& \multicolumn{2}{c}{\text { Gefunden }} & \text { Ber. für } \mathrm{C}_{4} \mathrm{H}_{7} \mathrm{NO}_{2} \\
& \text { I. } & \text { II. } & 13.86 \mathrm{pCt} .
\end{array}
$$


Schmelzpunkt und Siedepunkt des aus gewöhnlichern Allylalkohol bereiteten Esters liegen bei $21.5^{\circ}$ bezw. $203^{\circ}$, während für den anderen $22^{\circ}$ bezw. $204^{\circ}$ gefunden wurde.

Aus den mitgetheilten Beobachtungen in Verbindung mit den früheren von $\mathrm{Hübner}$ und Müller darf man wohl mit Sicherheit folgern, dass der aus symmetrisehem Dichlorhydrin und Natrium gebildete $A$ lkohol mit dem gewöhnlichen Allylalkohol identisch ist.

Meine weiteren Bestrebungen werden darauf gerichtet sein, durch modificirtes Verfahren die möglicherweise entstehenden intermediären Producte zu fassen, sowie durch ein näheres Studium des Verhaltens der Dichlorhydrine einen Einblick in die räthselhafte Reaction zu bekommen. Die in dieser Richtung angestellten Versuche sind noch nicht so weit vorgeschritten, dass über dieselben berichtet werden könnte.

Nur eine Beobachtung möchte ich gleich hicr erwähnen, die sich auf das Verhalten des bei $174.3^{\circ}$ siedenden Dichlorhydrins gegenüber Reductionsmitteln bezieht. Als Buff ${ }^{1}$ ) im Jahre 1867 das Dichlorhydrin, in wässerigem Aether gelöst, mit Natriumamalgam reducirte, war das unsymmetrische, bei $182^{\circ}$ siedende Dichlorhydrin noch nicht bekannt, welches meines Wissens zuerst einige Jahre später von Tollens ${ }^{2}$ ) dargestellt worden ist. Buff giebt weder den Siedepunkt seines Dichlorhydrins an, noch sagt er etwas von dem Verfahren, nach welchem er dasselbe bereitet hat. Wenn er dasselbe dureh fractionirte Destillation des aus Glycerin erbaltenen Rohproductes gewonnen hat, wird sein Präparat, späteren Angaben zufolge, ausser anderen Verunreinigungen jedenfalls auch etwas von dem unsymmetrischen Dichlorhydrin enthalten haben. Ws schien infolge dessen wünschenswerth, den von Buff gemachten Reductionsversuch zu wiederholen. Wider Erwarten bin ich zu einem anderen Resultat gelangt wie Buff, welches möglicherweise mit dem von Buff erbaltenen nicht unvereinbar ist, welches aber jedenfalls die von ihm aus seinem Versuche gemachten Folgerungen unzulässig erscheinen lässt.

Es wurde reines, aus Epichlorhydrin gewonnenes Dichlorhydrin in so viel wässerigem, alkoholfreiem Aether gelöst, dass etwa das Doppelte der sirforderlichen Menge Wassers vorhanden war, und dann mit etwas mehr als der berechneten Menge Natriumamalgam versetzt. Die Reaction verlief anfangs unter deutlich wahrnehmbarer Erwärmung, nach kurzer Zeit schien sie aber beendet zu sein, ohne dass die zur vollständigen Reduction erforderliche Menge Natriumamalgam zersetzt war. Die ätherische Lösung wurde darauf filtrirt, mit Potasche getrocknet und mittelst des Linnemann'schen Apparates der fractio-

1) Ann. Chem. Pharm. Supl. V, 247.

2) Ann. Chem. Pharm. 156, 164. 
nirten Destillation unterworfen. Aus etwa $8 \mathrm{~g}$ Dichlorhydrin bekam ich nur einige Tropfen einer zwischen 70 und $105^{\circ}$ übergehenden Fraction, welche unverkennbar nach Allylalkohol roch. Dagegen gewann ich gegen $4 \mathrm{~g}$ eines bei $114^{\circ}$ siedenden Oeles, welches Chlor enthielt. Das Oel wurde mit trockenem Salzsäuregas behandelt, wobei es sich stark erwärmte, so dass gekühlt werden musste. Als Endproduct resultirte eine syrupdicke Flüssigkeit, deren Siedepunkt mit dem des symmetrischen Dichlorhydrins $\left(174^{\circ}\right)$ übereinstimmte. Der Siedepunkt des Oeles (ich habe früher für Epichlorhydrin $113.8^{\circ}$ gefunden) sowie sein Verhalten gegenüber Salzsäure beweist genügend, dass dasselbe Epichlorhydrin gewesen ist. Zur Sicherheit habe ich den Versuch mit ganz frisch bereitetem fünfprocentigem Natriumamalgam wiederholt und dasselbe Resultat erhalten. Nachdem somit festgestellt war, dass sich bei der Reduction Epichlorbydrin bildet, bin ich, um die weitere Reduction zu verfolgen, vom Epichlorbydrin ausgegangen. $10 \mathrm{~g}$ Epichlorhydrin wurden mit wässerigem Aether und überschüssigem Natriumamalgam drei Tage in Berührung gelassen. Die Reduction verläuft sehr langsam, und es gelang mir nicht, die von Buff beobachtete Bildung von Isopropylalkohol nachzuweisen, vielmehr gewann ich die Hauptmenge des angewandten Epichlorhydrins wieder, und die wenigen Tropfen, die bei der fractionirten Destillation unter $105^{\circ}$ übergingen, rochen wiederum unverkennbar nach Allylalkohol. Es steht dies mit der Angabe von Müller und Tollens ${ }^{1)}$ in Einklang, die bei Reduction von symmetrisehem Dichlorhydrin mit Natriumamalgam Allylalkohol bekommen haben.

Es ist immerhin möglich, dass sich bei dieser Reduction anch Isopropylalkohol bildet, wenngleich ich denselben auch nicht nachzuweisen vermochte, jedenfalls geht die Reaction sehr schwer. Es ist aber sehr fraglich, ob es von Interesse für die Beurtheilung der Constitution der Dichlorhydrine ist, zu wissen, welches Product bei der Reduction schliesslich als Endproduct entsteht, nachdem mit Sicherheit nachgewiesen ist, dass Epichlorhydrin in grösseren Mengen als intermediäres Product auftritt. Das Epichlorhydrin muss nämlich aus dem symmetrischen Dichlorhydrin durch Salzäureabspaltung entstehen, und da bekanntermaassen auch das unsymmetrische, bei $182^{\circ}$ siedende Dichlorhydrin bei Chlorwasserstoffabspaltung Epichlorhydrin liefert, so werden wahrscheinlich alle beide Dichlorhydrine bei der Reduction mit Natriumamalgam in wässerigem Aether dieselben Producte geben.

Verschiedene Umstände zwingen mich, die weiteren Untersuchungen über dieses Thema vorläufig zu unterbrechen, doch hoffe ich, im Herbst dieselben wieder aufnehmen zu können.

1) Ann. Chem. Pharm. 159, 178. 
Die vorstehend beschriebénen Versuche sind im Göttinger Universitätslaboratorium ausgeführt worden, dessen Director, Hr. Prof. V. Meyer, mich bei denselben auf's Freundlichste unterstützt hat. Ich benutze gern die Gelegenheit, um demselben auch an dieser Stelle meinen herzlichsten Dank dafür abzustatten.

Göttingen. Univers.-Labor., im März 1888.

234. Victor Meyer: Ueber die negative Natur organischer Radicale und die Frage der Fxistenz wahrer Nitrosokörper.

\author{
(Eingegangen am 3. April.)
}

In zwei im vorigen Jahre veröffentlichten Abhandlungen: 》Ueber die negative Natur der Phenylgruppe ${ }^{1}$ ) und "Ueber die negative Natur organischer Radicale ${ }^{2}$ ) habe ich die Gesichtspunkte besprochen, welche für die in den folgenden Abhandlungen niedergelegten Arbeiten maassgebend gewesen sind. Dem schon damals Gesagten möchte ich heute nur wenige Bemerkungen hinzufügen.

Die bemerkenswerthe Thatsache, dass im Desoxybenzoïn - soweit die bisher angestellten Versuche es beurtheilen lassen - nur ein Methylenwasserstoffatom durch Alkyle ersetzbar ist, findet eine, aber nicht vollständig zutreffende Analogie in dem Verhalten des Benzylcyanids, welches, je nach den Umständen, zwei Radikale aufnimmt, oder aber dem Eintritte eines zweiten einen unüberwindlichen Widerstand entgegenstellt. Das Studium dieser höchst merkwürdigen Verhältnisse hat mich seit Monaten beschäftigt, ohne dass es mir bisher möglich gewesen wäre, die Gründe der auffallenden Erscheinung völlig aufzuklären. Die bezüglichen Untersuchungen setze ich indessen fort, und ich hoffe, bald über dieselben Näheres mittheilen zu können; über die zur Aufklärung dieses Punktes unternommenen Versuche ist in den folgenden Abhandlungen noch nichts enthalten.

Während ich früher auf die grosse Analogie hinwies, welche zwischen Malon- und Acetessigester einerseits, Desoxybenzoïn und Benz ylcy anid andererseits besteht, möchte ich heute eine charakteristische Verschiedenheit hervorheben. Die Natriumverbindungen des Desoxybenzoïns und Benzylcyanids, welche man bei den erwähnten Synthesen

1) Diese Berichte XX, 534.

2) Ib. 2944 .

Bexichte d. D. ehem. Gesellschaft. Jahrg. XXI, 